\title{
SET MEMBERSHIP ESTIMATION WITH A SEPARATE RESTRICTION ON INITIAL STATE AND DISTURBANCES ${ }^{1}$
}

\author{
Polina A. Yurovskikh \\ Krasovskii Institute of Mathematics and Mechanics, \\ Ural Branch of the Russian Academy of Sciences, \\ 16 S. Kovalevskaya Str., Ekaterinburg, 620108 Russia \\ polina2104@list.ru
}

\begin{abstract}
We consider a set membership estimation problem for linear non-stationary systems for which initial states belong to a compact set and uncertain disturbances in an observation equation are integrally restricted. We prove that the exact information set of the system can be approximated by a set of external ellipsoids in the absence of disturbances in the dynamic equation. There are three examples of linear systems. Two examples illustrate the main theorem of the paper, the latter one shows the possibility of generalizing the theorem to the case with disturbances in the dynamic equation.
\end{abstract}

Keywords: Set membership estimation, Filtration, Approximation, Information set, Ellipsoid approach.

\section{Introduction and notations}

Set membership approaches to estimation problems have been studied for a long time [3, 10]. In 1968, Krasovskii proposed [7], and later Kurzhanski developed [8, 9] a more general theory of guaranteed estimation without the statistics of disturbances based on results of convex and functional analysis.

This paper is an addition to [2] which describes the approximation of the estimation problem for joint constraints on the initial state and disturbances with the ellipsoid technique. In this case, an optimization problem arises. The paper considers a simpler case when the exact information set of the system can be found without solving an optimization problem. The technique of ellipsoidal approximation is used, which was developed by Kurzhanski [9], Chernousko [4], and their followers (see, for example, [6]).

The paper is structured as follows. First, we formulate the estimation problem in our case, then construct exact information sets and their approximation using external ellipsoids. After that, we prove the validity of the approximation. The latter part consists of three numerical examples.

Let us introduce the notation. Let

$$
|x|_{Q}=\sqrt{x^{\prime} Q x}
$$

where $x \in \mathbb{R}^{n}$ and $Q$ is a matrix with the property $Q^{\prime}=Q>0$. For $Q=I$ (an identity matrix), we set $|x|_{I}=|x|$. If $M \subset \mathbb{R}^{n}$ is convex and compact, then

$$
\rho(l \mid M)=\max _{x \in M} l^{\prime} x
$$

\footnotetext{
${ }^{1}$ This study is a part of the research carried out at the Ural Mathematical Center and supported by the Ministry of Science and Higher Education of the Russian Federation (agreement no. 075-02-2021-1383).
} 
is a support function. The set

$$
E(Q, c)=\left\{x \in \mathbb{R}^{n}|| x-\left.c\right|_{Q} \leqslant 1\right\}
$$

is called an ellipsoid.

If a system is linear and non-stationary, i.e., $\dot{x}=A(t) x$, then its general solution has the form $x\left(t, t_{0}, x_{0}\right)=\mathbf{X}\left(t, t_{0}\right) x_{0}$, where $\mathbf{X}\left(t, t_{0}\right)$ is a fundamental matrix, which can be found as a solution to the equation $\dot{\mathbf{X}}\left(t, t_{0}\right)=A(t) \mathbf{X}\left(t, t_{0}\right), \mathbf{X}\left(t_{0}, t_{0}\right)=I$.

\section{Problem statement}

Consider a linear non-stationary system with measurements

$$
\dot{x}=A(t) x, \quad y=G(t) x+w, \quad t \in[0, T],
$$

where $x(t) \in \mathbb{R}^{n}$ is a state vector, $y(t) \in \mathbb{R}^{m}$ is an output, $w(t) \in \mathbb{R}^{m}$ is an uncertain disturbance in the measurement equation, and $A(t) \in \mathbb{R}^{n \times n}$ and $G(t) \in \mathbb{R}^{m \times n}$ are bounded continuous matrices. Suppose that undefined functions $w(\cdot)$ in $(2.1)$ and an initial state $x_{0}$ satisfy the following integral and geometric constraints, respectively:

$$
\begin{gathered}
\int_{0}^{T}|w(t)|_{R}^{2} \leqslant 1, \quad R^{\prime}(t)=R(t)>0 \\
x_{0} \in X_{0}
\end{gathered}
$$

where $X_{0} \in \mathbb{R}^{n}$ is a convex compact set bounding the initial state, and $R(t) \in \mathbb{R}^{m \times m}$ is a continuous positive definite matrix. The constraints are separate, i.e., (2.2) and (2.3) are independent. According to the general theory of guaranteed estimation (see, for example, [9]) we can give a definition.

Definition 1. A family of state vectors $\mathcal{X}(T, y)=\left\{x_{T}\right\}$ is called an information set (IS) if, for any $x_{T} \in \mathcal{X}(T, y)$, there exists a function $w$ and an initial state $x_{0}$ satisfying constraints (2.2) and (2.3) and such that equalities (2.1) hold almost everywhere with $x(T)=x_{T}$.

For system (2.1) under constraints (2.2) and (2.3), an exact set $\mathcal{X}(T, y)$ can be found.

Theorem 1. The set $\mathcal{X}(T, y)$ is an intersection

$$
\mathcal{X}(T, y)=\mathbf{X}(T, 0) X_{0} \bigcap \mathbb{X}(T, y, 0,0)
$$

where $\mathbf{X}(T, s)$ is the fundamental matrix of system (2.1), $\mathbb{X}(T, y, 0,0)$ is the IS for (2.1) and (2.2) without constraints on the initial set (2.3).

Consider linear system (2.1) under constraint (2.2). A solution to the estimation problem is the IS $\mathbb{X}(T, y, 0,0)$, which is an ellipsoid $x^{\prime} P(T) x-2 x^{\prime} d(T)+q(T) \leqslant 1$ whose parameters can be found as solutions to the differential equations $[1,2]$

$$
\begin{gathered}
\dot{P}(t)=-A^{\prime}(t) P-P A(t)+G^{\prime} R G, \quad P(0)=0 ; \\
\dot{d}(t)=-A^{\prime}(t) d+G^{\prime} R y, \quad d(0)=0 ; \\
\dot{q}(t)=y^{\prime} R y, \quad q(0)=0 .
\end{gathered}
$$




\section{Approximation of information sets}

The original problem included integral constraints on perturbations (2.2) and geometric constraints on the initial state (2.3) of the system. Geometric constraints in form (2.3) are complicated to deal with. Kurzhanski proposed an approach for approximating arbitrary sets (see, for example, [9]) by sets of ellipsoids. In this paper, we discuss the approximation by a set of external ellipsoids.

We approximate the set of initial states $X_{0}$ by a family of ellipsoids $E\left(P_{0}, c\right) \supset X_{0}$, where $P_{0}$ is a symmetric positive definite matrix $P_{0}^{\prime}=P_{0}>0$. Then constraints $(2.2)$ and $(2.3)$ will be approximated by the family of constraints

$$
\alpha\left|x_{0}\right|_{P_{0}}^{2}+(1-\alpha) \int_{0}^{T}|w(t)|_{R}^{2} \leqslant 1, \quad \alpha \in[0,1] .
$$

Thus, we obtain the second estimation problem of (2.1) under constraints (3.1).

If disturbances $w(t)$ satisfy the constraint in (2.2), then they necessarily obey the constraints in (3.1). Therefore, it is possible to build an IS $\mathbb{X}\left(T, y, \alpha, P_{0}\right)$ for a real signal with different parameters and use it to approximate the original IS $\mathcal{X}(T, y)$.

Lemma 1. The set $\mathbb{X}\left(T, y, \alpha, P_{0}\right)$ has the form of an ellipsoid

$$
x^{\prime} P(T) x-2 x^{\prime} d(T)+q(T) \leqslant 1,
$$

where the parameters are defined as solutions to the differential equations [1, 2]

$$
\begin{gathered}
\dot{P}=-A^{\prime}(t) P-P A(t)+G^{\prime} R G(1-\alpha), \quad P(0)=P_{0} \alpha ; \\
\dot{d}=-A^{\prime}(t) d+G^{\prime} R y(t)(1-\alpha), \quad d(0)=0 ; \\
\dot{q}=y^{\prime}(t) R y(t)(1-\alpha), \quad q(0)=0 .
\end{gathered}
$$

Lemma 2 (Ellipsoid Separation Lemma). For every convex compact set $M \subset \mathbb{R}^{n}$ and a point $p \notin M$, there exist an ellipsoid $E(Q, c)$ such that $E(Q, c) \supset M$ and $p \notin E(Q, c)$.

P r o o f. It is known from convex analysis (see, for example, [5]), that the condition $p \notin M$ implies the existence of a unit vector $l_{1}$ such that $l_{1}^{\prime} p>\rho\left(l_{1} \mid M\right)$. Further, since the set $M$ is fixed, we use the shorter notation $\rho(l)$. Let us complement the vector $l_{1}$ to an orthonormal basis in $\mathbb{R}^{n}$ with vectors $\left\{l_{2}, \ldots, l_{n}\right\}$. Build a rectangular box along $l_{i}$ centered at the point

$$
c=\sum_{i=1}^{n} l_{i}\left(\rho\left(l_{i}\right)-\rho\left(-l_{i}\right)\right) / 2
$$

and having vertices at the points

$$
A_{k}=\sum_{i=1}^{n} k_{i} l_{i} \rho\left(k_{i} l_{i}\right): \Pi=\left\{x \in \mathbb{R}^{n} \mid \rho\left(-l_{i}\right) \leqslant l_{i}^{\prime} x \leqslant \rho\left(l_{i}\right) \forall i \in 1: n\right\} .
$$

Here, $k \in K \subset \mathbb{R}^{n}$ is a vector with coordinates $k_{i}= \pm 1$. The number of such vectors and vertices is $2 n$; the set $K$ contains all such vectors $k$. Let us arrange the set $K=\left\{k^{1}, \ldots, k^{2 n}\right\}$ assuming that $k^{1}=[1 ; \ldots ; 1]$. This box will contain the original compact set: $\Pi \supset M$.

We introduce an orthogonal matrix $T=\left[l_{1}, \ldots, l_{n}\right]$ and perform an orthogonal transformation to new coordinates $y=T^{\prime} x$. In the new coordinates, the set $M$ becomes $M^{*}=T^{\prime} M$, and the box $\Pi$ becomes the box $\Pi^{*}=T^{\prime} \Pi$ with center $c^{*}=T^{\prime} c$ and edges parallel to the coordinate axes. We 
have $l_{1}^{\prime} p=l_{1}^{\prime} T p=[1,0, \ldots, 0] p^{*}=p_{1}^{*}>\rho\left(l_{1}\right)$ by the condition. We build an ellipsoid with the center $c^{*}$ through the vertices of the box $A_{k}^{*}$ and axes parallel to the coordinate axes, consisting of vectors of the form $c^{*}+y$, where the coordinates of the vector $y$ satisfy the equation

$$
\sum_{i=1}^{n} y_{i}^{2} / b_{i}^{2}=1
$$

Denote by $a_{i}=\left(\rho\left(l_{i}\right)+\rho\left(-l_{i}\right)\right) / 2$ the box semiaxes. Let us choose the parameters $b_{i}$ of the ellipsoid so that

$$
\sum_{i=1}^{n} a_{i}^{2} / b_{i}^{2}=1, \quad \rho\left(l_{1}\right)<b_{1}+c_{1}^{*}<p_{1}^{*} .
$$

Since $\rho\left(l_{1}\right)-c_{1}^{*}=a_{1}<b_{1}$, the other $b_{i}$ can be taken equal to $a_{i}+t, i \in 2: n$, where $t$ can be found from the equation

$$
\sum_{i=2}^{n} a_{i}^{2} /\left(a_{i}+t\right)^{2}=1-a_{1}^{2} / b_{1}^{2}
$$

The obtained ellipsoid $E^{*}$ with conditions (3.3), (3.4), and (3.5) is such that $E^{*} \supset \Pi^{*} \supset M^{*}$ and $p^{*} \notin E^{*}$. We obtain the desired ellipsoid with the properties $E(Q, c) \supset \Pi \supset M$ and $p \notin E(Q, c)$ by performing the inverse transformation $x=T y$. Here, the matrix $Q=T \Lambda T^{\prime}$ and $\Lambda=\operatorname{diag}\left(1 / b_{1}, \ldots, 1 / b_{n}\right)$.

Remark 1 . If the set $M$ is centrally symmetric, then $c=0$.

Theorem 2. Let $X_{0}$ be a centrally symmetric set. Then the set $\mathcal{X}(T, y)$ is an intersection

$$
\mathcal{X}(T, y)=\bigcap_{P_{0} \in \mathcal{P}_{0}, \alpha} \mathbb{X}\left(T, y, P_{0}, \alpha\right)
$$

where $\mathcal{P}_{0}$ is a set of symmetric positive matrices $P_{0}$ such that $E\left(P_{0}, 0\right) \supset X_{0}$.

P r o o f. We construct the proof by contradiction. Consider the inclusion

$$
\mathcal{X}(T, y) \supset \bigcap_{P_{0} \in \mathcal{P}_{0}, \alpha} \mathbb{X}\left(T, y, P_{0}, \alpha\right) .
$$

Let

$$
x_{*} \in \bigcap_{P_{0} \in \mathcal{P}_{0}, \alpha} \mathbb{X}\left(T, y, P_{0}, \alpha\right),
$$

but $x_{*} \notin \mathcal{X}(T, y)$. Then either $x_{*} \notin \mathbb{X}(T, y, 0,0)$ or $x_{*} \notin \mathbf{X}(T, 0) X_{0}$. The first is impossible, since $\mathbb{X}(T, y, 0,0)$ is among $\mathbb{X}\left(T, y, P_{0}, \alpha\right)$ when the parameters $P_{0}=0$ and $\alpha=0$ are chosen. Consider the second possibility. If $x_{*} \notin \mathbf{X}(T, 0) X_{0}$ is true, then $x_{0}=\mathbf{X}(0, T) x_{*} \notin X_{0}$. By Lemma 2 and Remark 1, there exists an ellipsoid $E(Q, 0)$ containing $X_{0}$ but not containing $x_{0}$. There is also a parameter $\alpha$ such that $x_{*} \notin \mathbb{X}(T, y, Q, \alpha)$.

We get a contradiction, since the set includes only vectors $x_{*}$, for which $x_{0}^{\prime} Q x_{0} \leqslant 1$ and $x_{*} \in \mathbb{X}(T, y, 0,0)$. The embedding

$$
\bigcap_{P_{0} \in \mathcal{P}_{0}, \alpha} \mathbb{X}\left(T, y, P_{0}, \alpha\right) \supset \mathcal{X}(T, y)
$$

is obvious. 


\section{Numerical examples}

\subsection{Double integrator}

Consider the one-dimensional equations of motion of a material point

$$
\dot{x}^{1}=x^{2}, \quad \dot{x}^{2}=0 .
$$

The set of possible initial states is a square:

$$
X_{0}=\left\{x_{0}:\left|x_{0}^{1}\right| \leqslant 1,\left|x_{0}^{2}\right| \leqslant 1\right\} .
$$

The measurement $y(t)$ are related to the state vector via the observation equation

$$
y=x^{1}+w(t),
$$

where $w(t)$ is the measurement noise satisfying the integral constraint

$$
\int_{0}^{T} w^{2}(t) d t \leqslant 1
$$

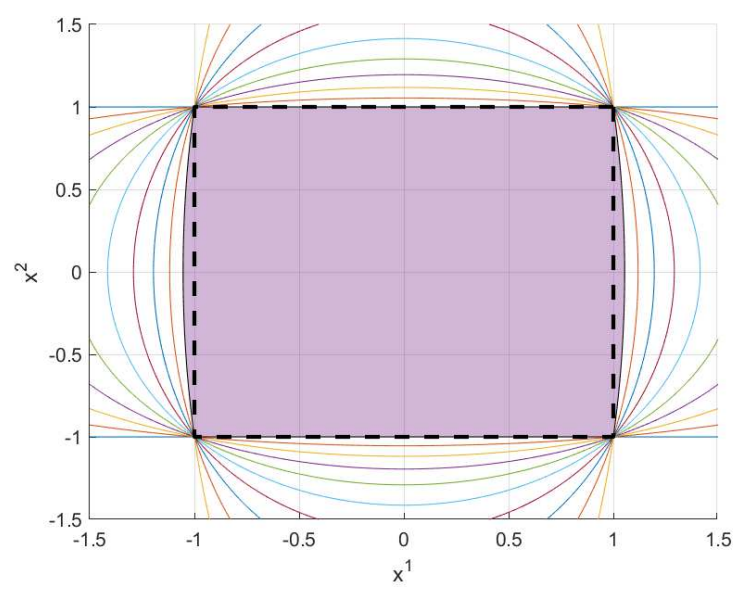

Figure 1. The set of possible initial states (black dashed line) and its approximation by external ellipses (pink fill).

Fig. 1 shows the approximation of the set of possible initial states by the intersection of a one-parameter family of ellipsoids with diagonal matrices $P_{0}=[a, 0 ; 0,1-a]$, where $a \in(0,1)$. The intersection of the family of ellipses does not perfectly approximate the set of initial states, which is a square; to avoid this, one should use degenerate ellipsoids. Then, each ellipse $\left\{x \mid x^{\prime} P_{0} x \leqslant 1\right\}$ will contain the square of initial states $X_{0}$, and their intersection will give an external approximation.

The parameters here are $G=[1,0], A=[0,1 ; 0,0]$, and $T=2$. For illustration, let us choose the signal generated by the admissible function $w(t)=0.8 \cos (t)$ and the admissible initial state $x_{0}=[1 ;-1] / 2$. Fig. 2 shows an approximation of the IS by a set of ellipsoids. The approximation of the IS (the white area on the left side of Fig. 2) coincides with the exact IS (the pink area on the right side of Fig. 2). The exact IS is obtained by the intersection of the reachable set at the terminal time (the black dashed line) and the IS without constraints on the initial state (the red dashed line). 

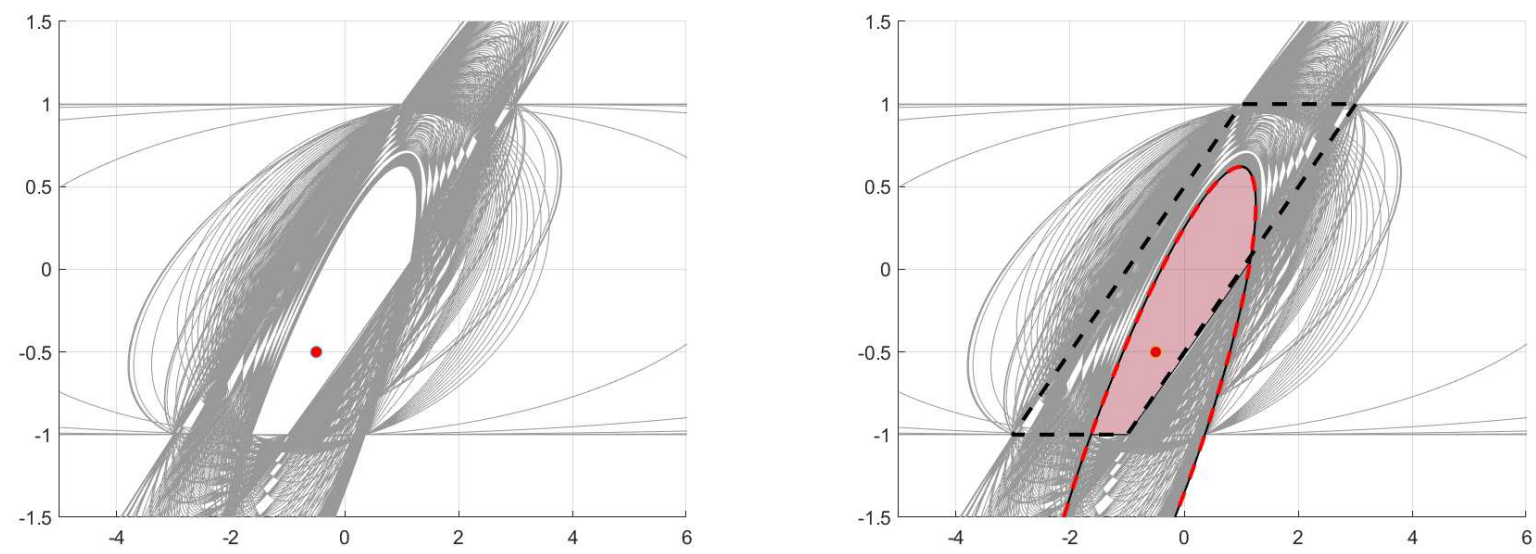

Figure 2. Double integrator. Approximation of the IS (on the left side) and the exact IS (on the right side). The red dot is the true state, the black dashed line is the reachable set at the terminal time $(T=2)$, and the red dashed line is the IS without constrains on the initial state.

\subsection{Mathematical pendulum}

Consider the equation

$$
\dot{x}^{1}=x^{2}, \quad \dot{x}^{2}=-25 x^{1} .
$$

The set of possible initial states is a circle: $X_{0}=\left\{x_{0}:\left|x_{0}\right| \leqslant 1\right\}$. The measurement equation is given by

$$
y=x^{1}+0.8 \cos (t)
$$

where $w(t)$ is the measurement noise, for which

$$
\int_{0}^{T}\left|y(t)-x^{1}(t)\right|^{2} d t \leqslant 1
$$

holds. The parameters here are $G=[1,0], A=[0,1 ;-25,0]$, and $T=2$. The implementation of disturbances and the initial state coincide with those in the previous example: $w(t)=0.8 \cos (t)$ and $x_{0}=[1 ; 1] / 2$.

\subsection{Double integrator II}

Consider a one-dimensional motion of a material point under disturbances $w^{1}(t)$ [2]:

$$
\dot{x}^{1}=x^{2}, \quad \dot{x}^{2}=w^{1}(t), \quad 0 \leqslant t \leqslant T .
$$

Let the disturbances $w^{1}$ also affect the measurement equation $y(t)=x^{1}(t)+w^{1}(t)+w^{2}(t)$, where $w^{2}$ is the measurement noise. Unfortunately, the calculation in [2] is inaccurate. Therefore, we need perform a new one. Define $w^{1}-w^{2}$ by $z(t)$. Since $w^{1}+w^{2}=y-x^{1}$, we obtain the following equations:

$$
\dot{x}^{1}=x^{2}, \quad \dot{x}^{2}=\left(y-x^{1}+z(t)\right) / 2 .
$$

The vector-valued function $w(t)$ subjects to the integral constraint $(2.2)$ with

$$
R=I_{2}, \quad \mathbf{V}=\mathbb{R}^{2}, \quad X_{0}=\left\{x \in \mathbb{R}^{2}|| x_{0}^{1}|\leqslant 1,| x_{0}^{2} \mid \leqslant 1\right\},
$$



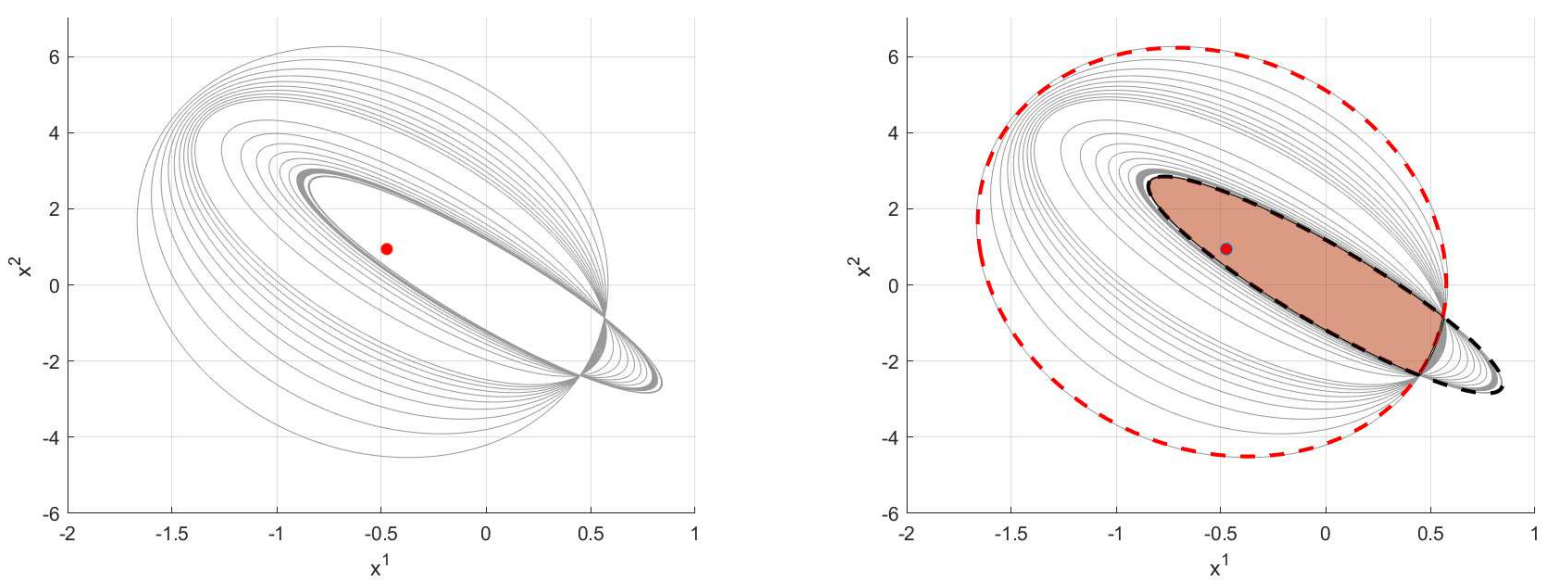

Figure 3. Mathematical pendulum. Approximation of the IS (left) and the exact IS (right). The red dot is the true state, the black dashed line is the reachable set at the terminal time $(T=2)$, and the red dashed line is the IS without constrains on the initial state.

i.e., this is the case of the absence of geometric constraints on $w(t)$. Since

$$
\left(w^{1}+w^{2}\right)^{2}+z^{2}=2|w|^{2}
$$

inequality (2.2) takes the form

$$
J\left(T, x_{T}, v, y\right)=\int_{0}^{T}\left(\left|y(t)-x^{1}(t)\right|^{2}+z^{2}(t)\right) d t / 2 \leqslant 1 .
$$

The constraints on initial states are the same as in the first example: a square is approximated by a one-parameter family of ellipses with diagonal matrices $P_{0}=[a, 0 ; 0,1-a]$, where $a \in(0,1)$. Then, each ellipse $\left\{x \mid x^{\prime} P_{0} x \leqslant 1\right\}$ contains the square of initial states $X_{0}$. Let is choose one more parameter $\alpha \in(0,1)$ and consider the constraints

$$
(1-\alpha)\left|x_{0}\right|_{P_{0}}^{2}+\alpha J\left(T, x_{T}, v, y\right)<1,
$$

where $J$ is defined in (4.2). The IS $\mathbf{X}_{T}^{a, \alpha}(y)$ for (4.1) under constrains (4.3) will contain the original IS $\mathbf{X}_{T}(y)$ for any signal in the original system. We will use relations (3.2). Then, we have

$$
\begin{gathered}
\mathbf{X}_{T}^{a, \alpha}(y)=\left\{x_{T}|| x_{T}-\left.\hat{x}(T)\right|_{P(T)} ^{2}+h(T)<1\right\}, \\
\dot{P}=-P(t) \tilde{A}-\tilde{A}^{\prime} P(t)+\alpha G^{\prime} G / 2-P(t) b C_{1} b^{\prime} P(t) / \alpha, \quad P(0)=(1-\alpha) P_{0} ; \\
\dot{\hat{x}}(t)=A \hat{x}(t)+\alpha\left(b c^{\prime}+P^{-1}(t) G^{\prime}\right)\left(y(t)-\hat{x}^{1}(t)\right) / 2, \\
\dot{h}(t)=\alpha\left|y(t)-\hat{x}^{1}(t)\right|^{2} / 2 .
\end{gathered}
$$

The parameters here are the same as in [2]: $b=[0,0 ; 1,0], c=[1,1], G=[1,0], C=1 / 2$, $C_{1}=[1,-1 ;-1,1] / 2$, and $\tilde{A}=[0,1 ;-0.5,0]$. We take the signal generated by the admissible functions $w^{1}(t)=0.8 \cos (t)$ and $w^{2}(t)=0.8 \sin (t)$ and the admissible initial state $x_{0}=[1 ;-1] / 2$.

\section{Conclusion}

The problem of estimating the state vector for a linear autonomous system under uncertainty has been solved. For such systems, the IS can be obtained as an intersection of ellipsoids. The third example shows that this can be also true for systems with disturbances in the dynamics equation. The issue will be considered in subsequent works. 


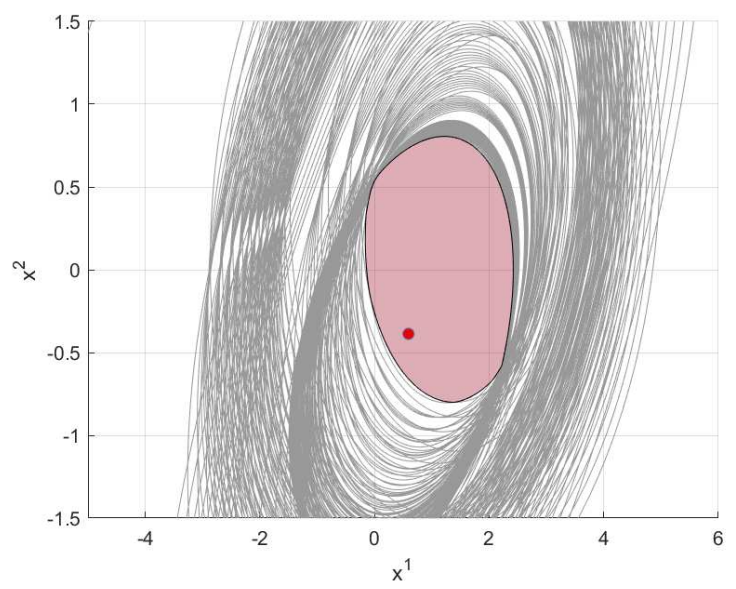

Figure 4. Double integrator II. The red dot is the true state at time $T=3$.

\section{REFERENCES}

1. Ananyev B. I., Yurovskih P. A. On the approximation of estimation problems for controlled systems. AIP Conf. Proc., 2019. Vol. 2164, No. 1. Art. no. 110001. P. 1-9. DOI: 10.1063/1.5130846

2. Ananyev B.I., Yurovskih P.A. Approximation of a guaranteed estimation problem with mixed constraints. Trudy Inst. Mat. Mekh. UrO RAN, 2020. T. 26, No. 4. P. 48-63. (in Russian) DOI: $10.21538 / 0134-4889-2020-26-4-48-63$

3. Bertsekas D. P., Rhodes I. B. Recursive state estimation for a set-membership description of uncertainty. IEEE Trans. Inform. Theory, 1971. Vol. 16, No. 2. P. 117-128. DOI: 10.1109/TAC.1971.1099674

4. Chernousko F. L. State Estimation for Dynamic Systems. CRC Press: Boca Raton, 1994. 320 p.

5. Dontchev A. L., Rockafellar R. T. Implicit Functions and Solution Mappings. 2nd ed. Springer Ser. Oper. Res. Financ. Eng. New York: Springer, 2014. 466 p. DOI: 10.1007/978-1-4939-1037-3

6. Filippova T.F. External estimates for reachable sets of a control system with uncertainty and combined nonlinearity. Proc. Steklov Inst. Math., 2018. Vol. 301, Suppl. 1. P. 32-43. DOI: $10.1134 /$ S0081543818050036

7. Krasovskii N. N. Teoriya upravleniya dvizheniem [Theory of Control of Motion]. Moscow: Nauka, 1968. 476 p. (in Russian)

8. Kurzhanski A. B. Upravlenie i nablyudenie v usloviyah neopredelennosti [Control and Observation under Conditions of Uncertainty]. Moscow: Nauka, 1977. 392 p. (in Russian)

9. Kurzhanski A. B., Varaiya P. Dynamics and Control of Trajectory Tubes: Theory and Computation. Systems Control Found. Appl., vol. 85. Basel: Birkhäuser, 2014. 445 p. DOI: 10.1007/978-3-319-10277-1

10. Schweppe F. C. Uncertain Dynamic Systems. New Jersey: Prentice-Hall, Englewood Cliffs, 1973. 563 p. 